\title{
Surgical Treatment of Dysembryoplastic Neuroepithelial Tumor
}

\author{
Guo Rongzeng ${ }^{1}$, Yang Zhongxu ${ }^{3,}$,, Cui Lihua ${ }^{1}$, You Zehong ${ }^{1}$, Du Ligong $^{1}$, Wang Yan $^{2}$, \\ Tang Zhensheng ${ }^{1}$ \\ ${ }^{1}$ Department of Functional Neurosurgery, Shanghai Blue Cross Brain Hospital, Shanghai, China \\ ${ }^{2}$ Center of Pathology, Fudan University, Shanghai, China \\ ${ }^{3}$ Department of Neurosurgery, the First Hospital of Tsinghua University, Beijing, China
}

Email address:

229028537@qq.com (Guo Rongzeng), zhongxuy67@163.com (Yang Zhongxu)

${ }^{*}$ Corresponding author

\section{To cite this article:}

Guo Rongzeng, Yang Zhongxu, Cui Lihua, You Zehong, Du Ligong, Wang Yan, Tang Zhensheng. Surgical Treatment of Dysembryoplastic Neuroepithelial Tumor. Advances in Surgical Sciences. Vol. 7, No. 1, 2019, pp. 9-14. doi: 10.11648/j.ass.20190701.13

Received: December 16, 2018; Accepted: January 3, 2019; Published: February 15, 2019

\begin{abstract}
Objective Investigating the surgical treatment for dysembryoplastic neuroepithelial tumor. Methods A total of 12 cases ( 7 males, 5 females, aged 4 36 years) of dysembryoplastic neuroepithelial tumors from 2010 to 2014 in Shanghai Blue Cross Brain Hospital were analyzed retrospectively, including 9 cases of temporal lobe tumor, 2 cases of frontal lobe tumor, and 1 case of parietal lobe tumor. The tumors and epileptogenic foci outside the lesions were resected under the guidance of electrocorticogram in all patients. Results The pathological examination showed that all the tumors were dysembryoplastic neuroepithelial tumors. All the patients had been followed up for 2 to 4 years. Epilepsy occurred in 2 patients and the others were seizure free after the surgery. No tumor recurrence occurred during the follow-up. Conclusion Dysembryoplastic neuroepithelial tumor is a benign tumor often causes intractable epilepsy, which can be controlled by the extended excision of the tumors and epileptogenic cortex under the guidance of electrocorticogram.
\end{abstract}

Keywords: Dysembryoplastic Neuroepithelial Tumors (DNET), Intractable Epilepsy, Electrocorticogram (ECoG), Surgery, Extended Resection

\section{Introduction}

Dysembryoplastic neuroepithelial tumours (DNET) are benign tumours of neuroglial origin and clinically characterized by early onset seizures. It is one of the most common surgical indications for epilepsy especially in younger age groups because of the favorable outcomes [1, 2], recurrent drug refractory epilepsy is the starting symptom in clinic, the tumor is similar to multiple nodular cortical tumor of semihamartoma [9, 10]. Radiological features are characterized in more detail and MRI subtypes were defined. Correlation of MRI subtypes and histopathological features were described in order to delineate the subtypes for more precise planning of surgery $[3,4,6]$. In this study, we retrospectively analysed the clinical, imaging and histopathological data of patients who were diagnosed with DNET and underwent surgery in our hospital. To analyze the relationship between clinical effect and surgical details [12, $13]$.

\section{Objects and Methods}

\subsection{General Information}

From August 2010 to August 2014, 12 cases of DNET patients were confirmed by pathology, including 7 males and 5 females, aged from 4 to 36 years old, and the history of the cases ranged from 6 months to 8 years. All the patients were medically refractory epilepsy.

\subsection{Clinical Manifestations}

Complex partial seizures were reported in 9 patients, tonic-clonus seizures in 3 patients, premonitory seizures in 5 patients, autonomic nerve symptoms in 2 patients, and panic attacks in 2 patients. The frequency of attack ranges from 2 
times/month to 4 5 times /day. All preoperative patients were treated with kinds of antiepileptic drugs (valproate sustained-release tablet $1000 \sim 2000 \mathrm{mg} /$ day, carbamazepine sustained-release tablets $400 \sim 1200 \mathrm{mg} / \mathrm{day}$, oxcarbazepine $1200 \sim 1800 \mathrm{mg} /$ day), some patients taking topiramate, lamotrigine, pyrazole raschig temples, phenytoin sodium in one or more, but all of the patients seizures $>2$ times/month, all the patients were characterized by a slow progression (there were more than two oral drugs, and the oral dose was increased, but the epilepsy could not be controlled. Five patients with a history of more than 5 years had cognitive declining). All the patients had no history of high fever and encephalitis, no history of postpartum asphyxia, no clear history of head trauma, and no family history of epilepsy.

\subsection{Preoperative Assessment}

The assessment included history inquiry, symptomatology description, CT/MRI examination, and video electroencephalogram (V-EEG) examination (at least $24 \mathrm{~h}$ for each examination) for all the cases for at least 2 times, sphenoid electrode examination was performed in 3 cases. MRI scan showed that the tumor was located in the temporal lobe in 9 cases (figure 1A, 1B), frontal lobe in 2 cases, and parietal lobe in 1 case. All the cases showed low signal on $\mathrm{T}_{1} \mathrm{WI}$ (figure $2 \mathrm{~A}, 2 \mathrm{~B}$ ), high signal on $\mathrm{T}_{2} \mathrm{WI}$ (figure $1 \mathrm{C}, 1 \mathrm{D}$ ), MRI flair imagine showed high signal, no enhancement, no obvious space-occupying effect, no obvious edema around the lesion, and 5 cases showed polycystic mixed signal. V-EEG examination showed spinous wave and spinal-slow wave in the lesion area in all cases (figure 4), sphenoid electrode tip wave in the lesion side in 3 cases, clinical attack was captured in 5 cases, and the initial lead location was consistent with the lesion area.

\subsection{Surgical Treatment}

Antiepileptic drugs were with drawed one day before surgery, but those with frequent epileptic seizures did not stop taking them. Phenobarbital sodium was prohibited before anesthesia. Tracheal intubation was used for general anesthesia, and laryngeal mask anesthesia was used for patients awakened during the operation. The surgical incision and exposure range were designed according to the tumor site and the abnormal discharge site of preoperative EEG monitoring, and the cortex and deep brain electrode monitoring (ECoG) were used during the operation. All 9 patients with temporal lobe epilepsy were treated with recumbent position, shoulder pad height, lateral cephalic position, frontal temporal flap craniotomy on one side, and the functional areas were protected by local anatomy (fissure of Sylvius, labe's vein) and the distance from the temporal pole to the resection boundary (left side $<5 \mathrm{~cm}$, right side $<6 \mathrm{~cm}$ ) to locate the resection range. Two patients with frontal lobe epilepsy and one patient with parietal lobe epilepsy underwent intraoperative arousal and cortical electrical stimulation to clarify the anatomical relationship between epileptic foci and functional areas, and then the surgical boundaries were determined. Neoplasm was found to be soft and dark gray with obvious boundaries between normal tissue and tumor. In 2 patients with frontal lobe epilepsy, the epileptic foci involved the lower part of the central anterior gyrus, and the contralateral oral and tongue movements were determined by cortical electrical stimulation; In 1 case of parietal lobe epilepsy, the epileptic foci involved the upper part of the central posterior gyrus, and abdominal discomfort was observed in patients with intraoperative arousal state by electrical stimulation of the cortex. All patients underwent complete resection of the tumor and epileptic foci, or low-power bipolar cortical thermal cauterization in the affected functional areas. Eclampsia discharge monitored by ECoG was located around the lesion in the operation. 9 cases of temporal lobe DNET patients underwent tumor plus hippocampal amygdala or standard anterior temporal lobectomy, and 2 cases of frontal lobe and 1 case of parietal lobe patients underwent low-power bipolarcortical thermal cauterization on the central cortex according to ECoG monitoring after tumor resection.

The average operation time was five hours, and the average intraoperative blood loss was $300 \mathrm{ml}$. There were no multiple lobectomy cases. The total resection rate of epileptic foci in this group was $75 \%$ ( 9 cases), low-power bipolar cortical thermal cauterization was performed in 3 cases $(25 \%)$ as the epileptic foci involved functional areas.

Postoperative fever is common ( 9 cases, accounting for $75 \%$ ), generally lasted for 3 to 5 days, the total number of white blood cells in blood examination and cerebrospinal fluid examination is normal, the proportion of neutral particles is normal or slightly increased. Considered for subarachnoid and ventricle system stimulation caused by inflammatory metabolites, after opening to give physical cooling and antipyretic analgesic drugs, The one who keeping at about $38.5^{\circ} \mathrm{C}$ temperature to give lumber pool catheter drainage, then the body temperature returned to normal within 1 week. No case of intracranial vascular injury, hemiplegia, aphasia, coma or death.

All surgical specimens were sent to the pathology center of Fudan University, and the pathological results of 12 patients were DNET, 9 patients had medial temporal lobe DNET together with hippocampal sclerosis, 2 patients had frontal lobe DNET and 1 patient had parietal lobe DNET with focal cortical dysplasia (FCD).

\section{Results}

Pathological diagnosis: Dysembryoplastic neuroepithelial tumor (WHO I level), the pathology report (figure 5a and 5b): tumor cell diffuse distribution, a tiny bursa, micro capsule floating among ganglion cells, tumor with branching small blood vessels, circle cell nucler, cytoplasm is empty, no obvious abnormity. Immunohistochemistry: tumor cells GFAP $(+)$, CD34 $($ focal +$)$, Nestin $(-/+)$.

All the patients were followed up for 2 to 4 years after surgery, with an average of 2.5 years. Electroencephalographies were reviewed at 3 months, 6 months, 1 year and 2 years after surgery: 6 cases of normal electroencephalogram, 4 cases of roughly 
normal electroencephalogram, and 2 cases of abnormal electroencephalogram. MRI examination reviewed at 2 years after surgery (figure 3 ) showed no tumor recurrence in all cases.

9 cases of patients with temporal lobe epilepsy and 1 case of frontal lobe epilepsy patients with postoperative follow-up period without any form of seizures, seizure control to Engel I level, the other 2 patients with abnormal existence central discharge line cortex hot burning technique but there is still a seizure, 1 case of frontal lobe epilepsy characterized by the contralateral limb automatism, 1 patient with parietal lobe epilepsy show the contralateral limb tonic-closure seizures, attacks the preoperative reduction, seizure frequency reduced more than $50 \%$, without conscious loss; According to Engel efficacy grading, 10 cases were cured $(83.3 \%)$ and 2 cases were significantly improved $(16.7 \%)$.

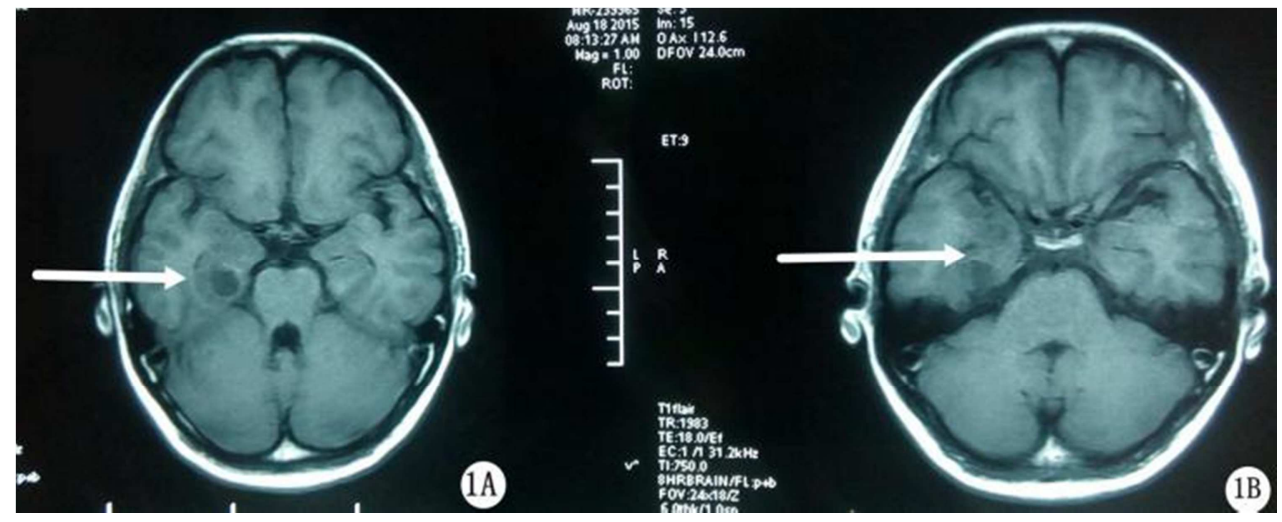

A and B: The tumor showed a polycystic, low signal intensity on T1WI, no tumor occupying effect.

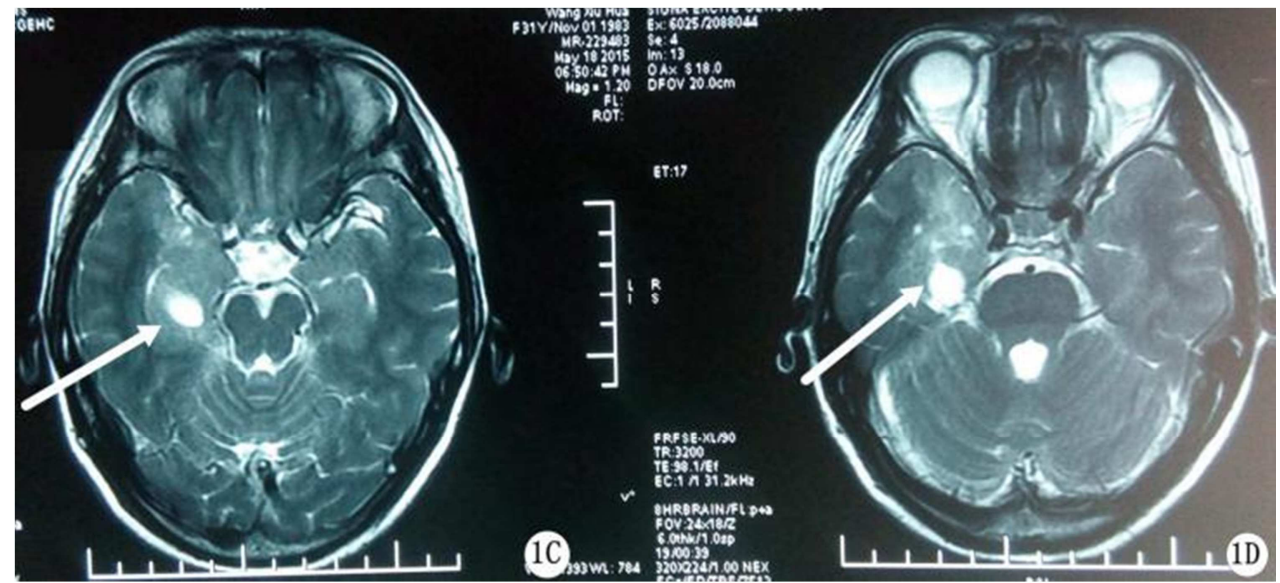

$\mathrm{C}$ and $\mathrm{D}$ : The tumor showed a mixed signal intensity on $\mathrm{T}_{2} \mathrm{WI}$, cystic degeneration has been seen in the tumor.

Figure 1. Preoperative plain MRI.

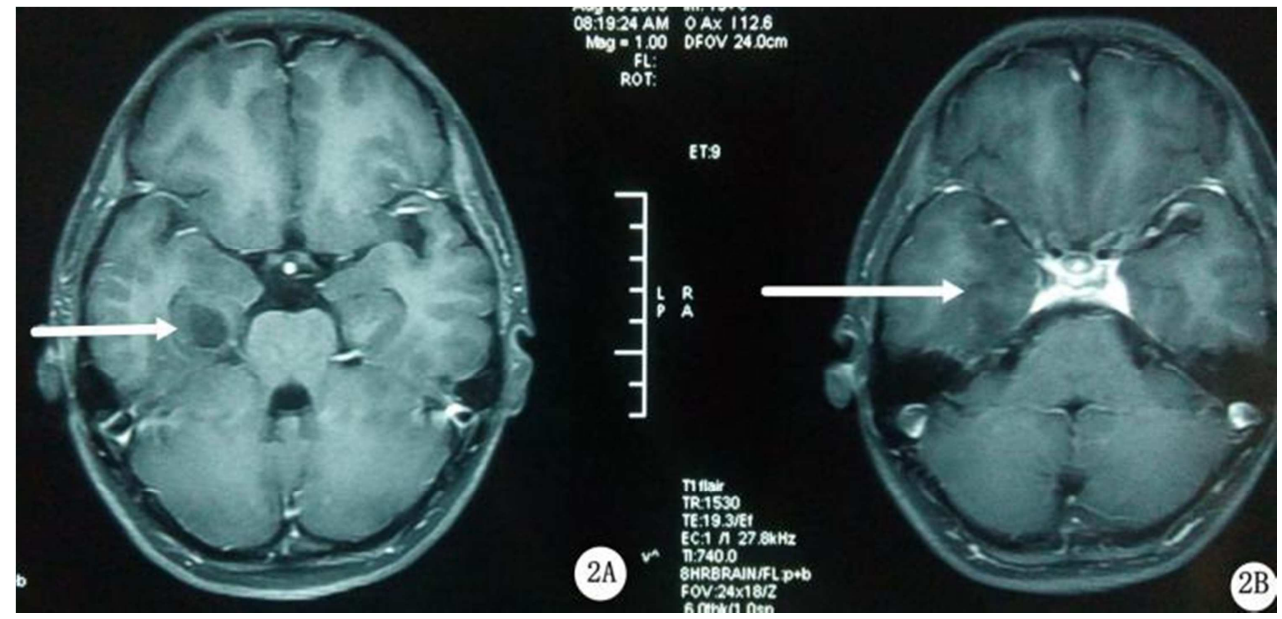

A and B: The image was transverse $\mathrm{T}_{1} \mathrm{WI}$ of contrast enhancement, which showed no enhancement of the tumor.

Figure 2. Contrast enhancement MRI. 


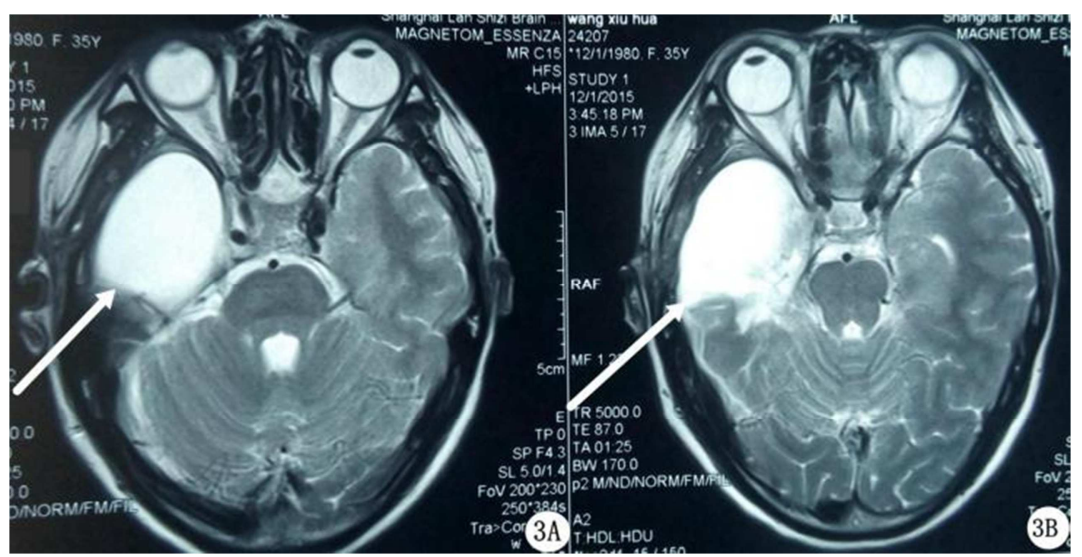

Figure 3. Postoperative 2 weeks MRI.

A: Standard anterior temporal lobectomy, the back boundary is located in front of the Labbe's vein; B: The tumor and the medial temporal structures were both removed.

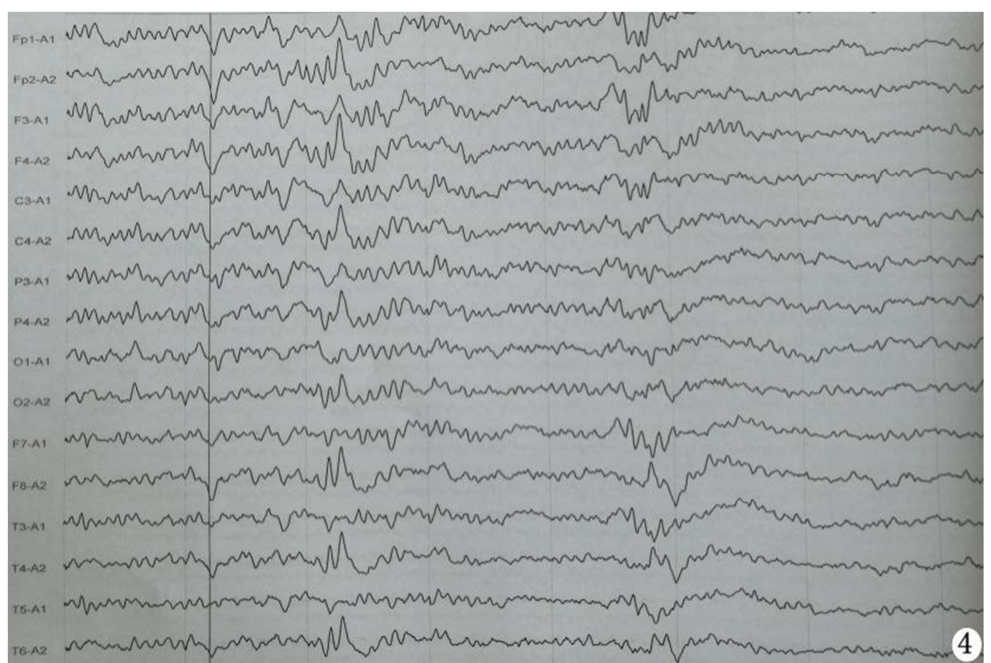

Figure 4. Preoperative V-EEG.

Median and high spike wave in the right frontal region and temporal region, spike and slow wave synchronous single shot, transient spike wave on the right side.
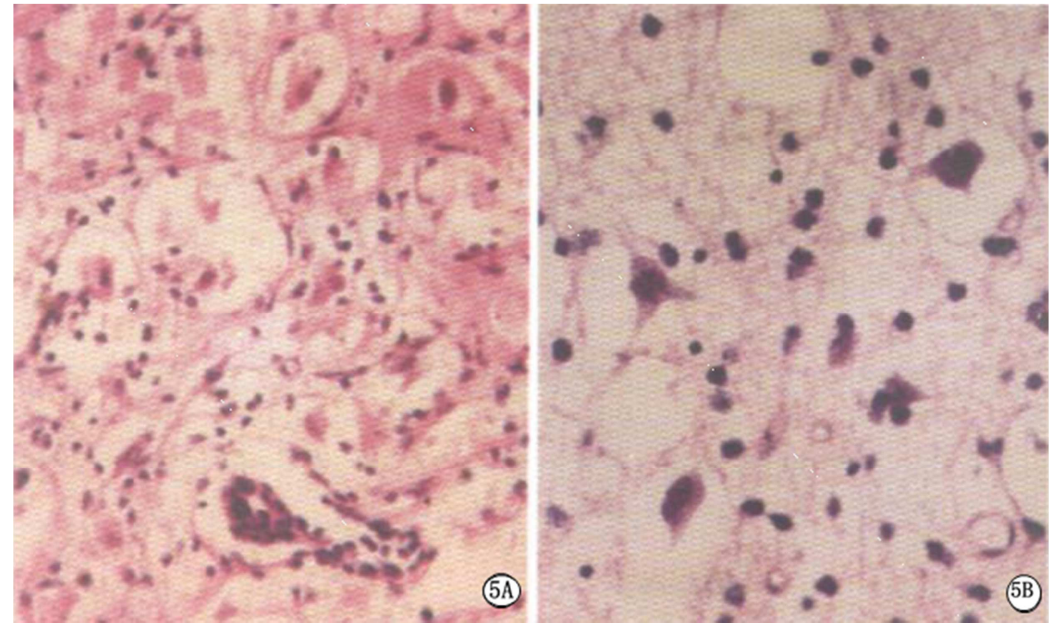

Figure 5. Microscopic cytologic examination: low and medium magnification.

A: Neuron-like cells scattered in between oligodendrocytes, medial cortical structural dysplasia $(\mathrm{HE}, \times 100)$. B: Neuron-like cells floating in microcapsules in the mucus background $(\mathrm{HE}, \times 200)$. 


\section{Disscusion}

French pathologist Daumas-Dupport and other scholars first reported 39 cases of neuroepithelial tumors in Neurosurgery in 1988, The neuroepithelial tumors, which are surgically curable and have independent histological characteristics, are all located in the supratentorial stage, presenting multi-nodular structures with different cellular components composed of astrocytes, oligodendrocytes and normal neurons [1]. CT scan showed low-density lesions with clear boundaries, often accompanied by abnormal cortical development at the lesion site. Therefore, it was speculated that the tumor was generated in the embryonic stage of cortical formation, possibly originated from submucosal cells, and was thus named as dysembryoplastic neuroepithelial tumor (DNET).

Dysembryoplastic neuroepithelial tumor (DNET) is mainly occured among adolescents [2], with more males than females. In this group, there were 12 patients, with an average age of 14 years, the largest of 36 years, and the longest history of 8 years. The lesions located in the temporal lobe accounted for $75 \%$ of the patients, and the clinical manifestations were long-term intractable and refractory epilepsy. Almost all the cases in this group presented as complex partial seizures, and the number of seizures increased with the progression of the disease course.

In the imaging manifestations of DNET [3, 4], the plain CT scan showed low/equal/high density signals, calcification and true cysts in a few cases, accompanied by skull deformity. MRI scan showed: $\mathrm{T}_{1}$-weighted low density, $\mathrm{T}_{2}$-weighted high density, accompanied by local cortical thickening. In the enhanced MRI/CT, about $1 / 3$ of the lesions showed annular enhancement, but there was no edema zone around the lesion and no space occupying effect. In the postoperative follow-up of MRI/CT in this study, the lesion was stable. Even if the lesion could not be completely resected during surgery, there was no imaging recurrence during the long-term postoperative follow-up, which was similar to the literature study.

DNET pathological examination [5-8] showed that the tumor was composed of oligodendroglioid cells, astrocytes and neurons. The boundary between the tumor tissue and brain tissue was clear, and there was no mitosis or necrosis. Neurons and astrocytes are mixed and float in the mucous matrix. Cystic changes are more common. This group of patients with tumor around or adjacent areas suffer from FCD and existing imaging techniques FCD surrounding the tumor may be difficult to find, but the postoperative pathological brain gray matter visible boundary is not clear, part of the cortex to arrange building disorder. FCD is type I - II according to the Plmini pathology classification model, and is common among patients with DNET, the incidence was $100 \%$. FCD is the focal point of eclampsia, so patients with DNET need to remove the surrounding dysplasia when removing the tumor.

ECoG monitoring was routinely used in DNET resection
[9], and it was found there were epileptic lesions around the site, including the irritation area and the onset area. Complete resection of the surrounding dysplasia structure while resection of the tumor was the key to ensuring the surgical efficacy. All 12 cases, 9 cases located in the temporal lobe in patients with tumor in the whole line of the hippocampus at the same time the amygdala or anterior temporal lobe resection, 9 cases of patients with postoperative seizure control to Engel I level, 1 case in the frontal lobe and 1 case in patients with abnormal existence central discharge line of the parietal cortex, low-power bipolar cortical thermal cauterization the cotex but there is still a postoperative seizures. The reason was that the focal dysplasia cortex in the functional area was not completely resected [10], and local abnormal electrical activity could still lead to epileptic seizures after the formation of spinous rhythm. Therefore, focal cerebral cortical dysplasia, hippocampal sclerosis and tumors are all epileptic foci, which should be removed surgically if possible [11-12].

DNET should be actively treated by surgery, and dynamic observation is not recommended. DNET is a static lesion, and radiotherapy and chemotherapy are not required after surgical resection of the tumor. DNET lesions total resection and epileptogenic zone surrounding the processing is very important to the postoperative seizure control [13-15], thus intraoperative $\mathrm{ECoG}$ monitoring should be regularly peformed, without damage to important brain function downward expanding excision, surrounding structures for the ribbon lesions, feasible to expand, including tumor resection, and residual lesions in functional areas, but the tumor excision of cerebral cortex after low-power bipolar cortical thermal cauterization, make the patients get good postoperative seizure control [16].

\section{Conclusion}

As a benign lesion, DNET has a good prognosis. It should be actively treated after diagnosis, postoperative radiotherapy and chemotherapy are not required. The patients in this group were followed up for 2.5 years on average postoperatively without recurrence or malignant transformation.

For DNET patients with epilepsy history, we advocate early surgical treatment, ECoG monitoring should be performed routinely during the operation, and the treatment of epileptic foci around pathological foci is crucial to the control of postoperative epileptic seizures, so as to achieve good epilepsy control. The epileptic foci involving functional areas cannot be directly resected and low-power bipolar cortical thermal cauterization can be performed.

For DNET patients with intractable epilepsy in the medial temporal lobe, temporal resection should be added on the basis of lesion resection medial lobectomy or anterior temporal lobectomy. 


\section{References}

[1] Meng Fangang, Sun Zhenrong, Zhang Jianguo, et al. Diagnosis and treatment of neuroepithelial tumors with embryonic dysplasia [J]. Chinese journal of neurosurgery, 2010, $26(3): 231-234$.

[2] Li Ling, Shu Kai, Lei Ting, et al. Pediatric epilepsy surgery [M]. 1st edition. Beijing: people's health publishing house, 2014:291-295.

[3] Yang Shujie, wang Dandan, Wang Ying, et al. MRI manifestations of embryonic dysplasia neuroepithelioma [J]. Journal of medical imaging, 2011, 21 (7) 967-970.

[4] Isler C, Erturk Cetin O, Ugurlar D, et al. Dysembryoplastic neuroepithelial tumours: clinical, radiological, pathological features and outcome [J]. Br J Neurosurg 2018 Aug; 32 (4): 436-441.

[5] Chen Li, Xu QingZhong, Pu YueShan et al. Neuroepithelial neoplasm and cortical dysplasia due to embryonic dysplasia [J]. Chinese journal of pathology, 2007, 36 (8):524-528.

[6] Yu Kai, Yu Shizhu, Zhang Jianning, et al. Clinical and pathological analysis of neuroepithelial neoplasm with embryonic dysplasia [J]. Chinese journal of neurosurgery, 2006, 22 (4):208-211.

[7] Chen Li, Lu Dehong, Xu Qingzhong. Clinicopathological observation of 11 cases of neuroepithelial neoplasm with embryonic dysplasia [J]. Chin J neurosci, 2006, 39 (8):525-528.

[8] Moazzam Alan A, Wagle Naveed, Shiroishi, et al. Malignant transformation of DNETs: a case report and literature review [J]. Neuroreport. 2014V25N12:894-9.

[9] Fallah A, Weil AG, Sur S, et al. Epilepsy surgery related to pediatric brain tumors: Miami Children's Hospital experience [J]. J Neurosurg Pediatr. 2015 Dec, 16 (6):675-80.

[10] Minkin K, Klein O, Mancini J, et al. Surgical strategies and seizure control in pediatric patients with dysembryoplastic neuroepithelial tumors: a single institution experience [J]. J Neurosurg Pediatrics, 2008, 1 (3):206-210.

[11] Daumas Dupport C, Scheithauer BW, Chodkiewicz JP, et al. Dsembryoplastic neuroepithelial tumor:a surgically curable tumor of young patients with intractable partial seizures. Report of thirty-nine cases [J]. Neurosurgery, 1988, 23 (7):545-556.

[12] Engel J Jr, Clinical neurophysiology, neuroimaging, and the surgical treatment of epilepsy [J]. Curr Opin Neurol Neurosurg, 1993, 6 (3):240-249.

[13] Nolan MA, Sakuta R, Chuang N, et, al. Dysembryoplastic neuroepithelial tumors in childhood: long-term outcome and prognostic features [J]. Neurology, 2004, 62 (4):2270-2276.

[14] Vogt VL, Witt JA, Delev D, et al. Cognitive features and surgical outcome of patients with long-term epilepsy-associated tumors within the temporal lobe [J]. Journal of epilepsy Behav. 2018 Nov, 88:25-32.

[15] Englot DJ, Berger MS, Barbaro NM, et al. Factors associated with seizure freedom in the surgical resection of glioneuronal tumors [J]. Epilepsia, 2012, 53 (1):51-57.

[16] Gao Xiang, Wang Yin, Jiang Chengchuan, et al. Diagnosis and treatment of neuroepithelioma with embryonic dysplasia $[\mathrm{J}]$. Chinese journal of neurosurgical diseases, 2004 (02):145-148. 